\title{
The sources of pharmaceuticals for problematic users of benzodiazepines and prescription opioids
}

\section{Suzanne Nielsen BPharm, BPharmSc(Hons), PhD, NHMRC Early Caree Research Fellow \\ Raimondo Bruno}

Senior Lecturer

Louisa Degenhardt BA(Hons), MClinPsych, PhD, Professor and NHMRC Principal Research Fellow ${ }^{3}$

Mark A Stoove PhD, BAAppSci(Hons) Head, ${ }^{4}$ and Senior Research Fellow

Jane A Fischer BA, MSPD, Senior Research Officer

Susan J Carruthers $\mathrm{MPH}, \mathrm{PhD}$

Research Fellow

Nicholas Lintzeris MB BS, FAChAM, PhD Director, ${ }^{8}$ and Clinical Associate Professor, ${ }^{9}$

1 Discipline of Addiction Medicine University of Sydney,

Sydney, NSW.

2 School of Psychology, University of Tasmania Hobart, TAS.

3 National Drug and Alcoho Research Centre, University of New South Wales Sydney, NSW.

4 HIV Research Program and Justice Health Research

Program, Centre for

Population Health

Burnet Institute,

Melbourne, VIC

5 Department of Epidemiology and

Preventive Medicine

Monash University.

Melbourne, VIC

6 National Centre for Education and

Training on Addiction,

Flinders University,

Adelaide, SA.

7 National Drug Research Institute, Curtin University,

Perth, WA.

8 Drug and

Alcohol Services,

South Eastern Sydney

Local Health District.

Sydney, NSW.

9 Faculty of Medicine,

University of Sydney,

Sydney, NSW.

suzanne.nielsen@

sydney.edu.au

MJA 2013; 199: 696-699 doi: 10.5694/mjal2.11331

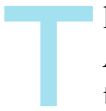

here are growing concerns in Australia and overseas about the increasing misuse of a range of pharmaceuticals, and associated harms. ${ }^{1-4}$ This includes the problematic use of benzodiazepines and prescription opioids in Australia.

Clients of drug treatment services are commonly reported to use a range of pharmaceuticals. ${ }^{5-7}$ For example, self-reported prevalence of benzodiazepine use among clients undergoing treatment for opioid use ranges from $66 \%$ to $100 \%, 5,7,8$ and use confirmed by urine drug testing ranges from $51 \%$ to $70 \% .^{8}$ Moreover, benzodiazepine use is associated with poorer treatment and health outcomes among opioid users. ${ }^{9,10}$ In North America, prescription opioiddependent clients outnumber heroindependent clients among those presenting for methadone maintenance treatment. ${ }^{6,11}$ Given the considerable harms that have been linked to these pharmaceuticals, including overdose ${ }^{12}$ and mortality, ${ }^{13,14}$ understanding their use and sources is important for informing effective harm-reduction interventions.

To our knowledge, no studies examining the sources of pharmaceuticals used by problematic users in Australia have been published, but sources of prescription opioids have been described in the United States. Among recent methadone treatment entrants who reported prescription opioids as the primary problem drug, the most common sources were dealers $(86 \%)$, friends and relatives (54\%) and medical prescriptions (28\%). ${ }^{6}$ In contrast, the US National Survey on Drug Use and Health found most non-medical users sourced analgesics from a friend or relative for free $(55 \%)$ or for payment $(11 \%){ }^{15}$ In both studies, the internet was rarely a cited source. 6,15

The Australian Government recently announced the introduction of a real-time reporting system for prescription opioids that doctors can use to obtain an up-to-date prescrip-

Objectives: To describe benzodiazepine and prescription opioid use by clients of drug treatment services and the sources of pharmaceuticals they use.

Design: Structured face-to-face interviews on unsanctioned use of benzodiazepines and prescription opioids were conducted between January and July 2008.

Participants: Convenience sample of treatment entrants who reported regular (an average of $\geqslant 4$ days per week) and unsanctioned use of benzodiazepines and/or prescription opioids over the 4 weeks before treatment entry.

Setting: Drug treatment services in Victoria, Queensland, Western Australia and Tasmania.

Main outcome measures: Participant demographics, characteristics of recent substance use, substance use trajectories, and sources of pharmaceuticals.

Results: Two hundred and four treatment entrants were interviewed. Prescription opioids were predominantly obtained from non-prescribed sources (78\%, 84/108). In contrast, medical practitioners were the main source for benzodiazepines (78\%, 113/144). Forging of prescriptions was extremely uncommon. A mean duration of 6.3 years (SD, 6.6 years) for benzodiazepines and 4.4 years (SD, 5.7 years) for prescription opioids was reported between first use and problematic use - a substantial window for intervention.

Conclusions: Medical practitioners are an important source of misused pharmaceuticals, but they are not the main source of prescription opioids. This has implications for prescription drug monitoring in Australia: current plans (to monitor only Schedule 8 benzodiazepines and prescription opioids) may have limited effects on prescription opioid users who use non-prescribed sources, and the omission of most benzodiazepines from monitoring programs may represent a lost opportunity for reducing unsanctioned use of benzodiazepines and associated harm.

tion history before prescribing drugs of addiction to any patient. ${ }^{16}$ It is planned that the reporting system will monitor Schedule 8 (S8) medications, therefore it will only include benzodiazepines that are S8. One benzodiazepine, flunitrazepam, is currently S8. Alprazolam will be rescheduled to S8 in 2014. To gauge the potential usefulness of such a system, it is important to understand how often these medications are being sourced through medical practitioners.

We describe (i) the characteristics of pharmaceutical use by Australian clients of drug treatment services who reported regular and unsanctioned use of benzodiazepines and/or prescription opioids and (ii) the common sources of benzodiazepines and prescription opioids used by these clients.

\section{Methods}

Detailed methods are available elsewhere. ${ }^{17}$ In brief, we recruited a con- venience sample of 305 people entering treatment at private and public withdrawal (detoxification), residential rehabilitation and opioid substitution treatment services in Victoria, Western Australia, Queensland and Tasmania. They were recruited between January and July 2008 by referral from treatment service staff and using study flyers that were distributed from drug treatment services by the staff.

Treatment entrants were eligible to participate in the study if they: reported regular (an average of $\geqslant 4$ days per week) and unsanctioned use of benzodiazepines and/or prescription opioids over the 4 weeks before treatment entry; were 16 years or older; had entered treatment for drug or alcohol use in the previous 6 months; and had sufficient English language skills to provide informed consent and comprehend the study questions. Unsanctioned use was defined as using medication that was 
not prescribed to the participant or using medication that was prescribed to the participant but in a way not intended by the prescriber (eg, taking a higher dose than prescribed, or injecting a drug that was intended as oral medication).

Structured face-to-face interviews of participants were conducted. They included questions on demographics (sex, age, ethnicity, employment, education level attained, current living arrangements), substance use (primary drug of concern at treatment entry, use in the 4 weeks before treatment entry, routes of administration), trajectories of substance use, and benzodiazepine and prescription opioid sources).

We used $\chi^{2}$ and $t$ tests to establish whether there were differences between participants who used benzodiazepines and those who used prescription opioids. Descriptive analyses were used to characterise benzodiazepine and prescription opioid initiation, use and sources.

The study was approved by institutional ethics committees in each jurisdiction - the Victorian Department of Human Services Human Research Ethics Committee, University of Tasmania Social Sciences Human Research Ethics Committee, Curtin University Human Research Ethics Committee and Prince Charles Hospital Human Research Ethics Committee.

\section{Results}

\section{Characteristics of participants}

A total of 204 people participated in the study; 107 had entered methadone and buprenorphine treatment programs, and the remainder had entered inpatient detoxification and residential rehabilitation programs (Box 1). One hundred and forty-two participants $(70 \%)$ were seeking treatment for opioid dependence (heroin or pharmaceutical opioids), 19 (9\%) were seeking treatment for benzodiazepine dependence, and 43 (21\%) were seeking treatment for dependence on other substances (eg, alcohol, stimulants and cannabis). When asked about substance use in the 4 weeks before treatment entry, 144 participants reported regular and unsanctioned benzodiazepine use and 108 reported regular and unsanc- tioned prescription opioid use; 48 reported regular and unsanctioned use of both drug classes.

The demographic characteristics of participants who used benzodiazepines only, prescription opioids only and both benzodiazepines and prescription opioids were similar about $60 \%$ men, mean age about 33 years, and low levels of employment and education.

Of the 144 participants who met the criteria for regular unsanctioned benzodiazepine use (with or without prescription opioids), 18 (13\%) reported benzodiazepines as their primary drug of concern, and 108 (75\%) reported that their benzodiazepine use had become problematic. In contrast, more than half $(56 \%, 60)$ of the 108 participants who met the criteria for regular unsanctioned prescription opioid use (with or without benzodiazepines) reported prescription opioids as their primary drug of concern, and 93 (86\%) reported that their prescription opioid use had become problematic.

\section{Pharmaceutical use in the 28 days before treatment entry}

On average, benzodiazepines and prescription opioids were used almost daily (27 out of 28 days for both) (Box $2)$. The benzodiazepines most fre-
1 Characteristics of the study participants $(n=204)$

\begin{tabular}{lc} 
& Number (\%)* \\
\hline Men & $126(62 \%)$ \\
Mean (SD) age, years & $32.6(8.7)$ \\
Aboriginal or Torres Strait Islander & $14(7 \%)$ \\
Employed full time or part time & $40(20 \%)$ \\
Completed up to year ll equivalent or less & $126(62 \%)$ \\
Living in unstable housing & $59(29 \%)$ \\
Current treatment & \\
Methadone & $64(31 \%)$ \\
Buprenorphine (with or without naloxone) & $43(21 \%)$ \\
Inpatient detoxification & $49(24 \%)$ \\
Residential rehabilitation & $48(24 \%)$ \\
Regular and unsanctioned substance use & \\
Benzodiazepines only & $96(47 \%)$ \\
Pharmaceutical opioids only & $60(29 \%)$ \\
Benzodiazepines and prescription opioids & $48(24 \%)$ \\
\hline * Data are number (\%) unless otherwise specified.
\end{tabular}

quently used as prescribed were diazepam (36\%, 52/144), alprazolam $(13 \%, 19 / 144)$ and oxazepam $(13 \%$, 18/144). For unsanctioned use, the benzodiazepines used most frequently were diazepam (80\%, 115/ $144)$, alprazolam $(58 \%, 84 / 144)$ and oxazepam $(44 \%, 63 / 144)$. The prescription opioids most frequently used as prescribed were morphine preparations $(10 \%, 11 / 108)$ and buprenorphine-naloxone tablets $(8 \%, 9 / 108)$. For unsanctioned use, the prescrip-

\section{Characteristics of benzodiazepine and prescription opioid use by study participants}

\begin{tabular}{|c|c|c|}
\hline & $\begin{array}{l}\text { Benzodiazepine } \\
\text { users }(n=144)\end{array}$ & $\begin{array}{l}\text { Prescription opioid } \\
\text { users }(n=108)\end{array}$ \\
\hline \multicolumn{3}{|l|}{ Use in 28 days before treatment entry } \\
\hline Mean (SD) period of prescribed use, days & $12.5(13.0)$ & $7.5(11.4)$ \\
\hline Mean (SD) period of non-prescribed* oral use, days & $20.9(9.6)$ & $16.3(12.2)$ \\
\hline Mean (SD) period of injected use, days & $0.9(4.2)$ & $16.5(12.0)$ \\
\hline Mean (SD) period of use in any form, days & $27.3(2.3)$ & $26.9(2.8)$ \\
\hline \multicolumn{3}{|l|}{ First-use occasion } \\
\hline Medication prescribed on first-use occasion, number (\%) & $41(28 \%)$ & $27(25 \%)$ \\
\hline \multicolumn{3}{|l|}{ Trajectory of use $^{\dagger}$} \\
\hline Mean (SD) age at first use, years & $18.9(6.9)$ & $21.2(8.1)$ \\
\hline Mean (SD) age when first injected, years & $22.7(7.6)$ & $22.7(7.8)$ \\
\hline Mean (SD) time from first use to monthly use, years & $4.1(5.9)$ & $3.0(5.1)$ \\
\hline Mean (SD) time from first use to problematic use, years & $6.3(6.6)$ & $4.4(5.7)$ \\
\hline \multicolumn{3}{|l|}{ Last use } \\
\hline Medication used was drug of choice, number (\%) & $50(35 \%)$ & $71(66 \%)$ \\
\hline \multicolumn{3}{|l|}{ Medication used was not drug of choice, number (\%) } \\
\hline Seeking intoxication & $29(20 \%)$ & $10(9 \%)$ \\
\hline Used to avoid or treat withdrawal symptoms & $19(13 \%)$ & $1(1 \%)$ \\
\hline Used as substitute for heroin or other opioids & na & $14(13 \%)$ \\
\hline Used for pain relief & na & $4(4 \%)$ \\
\hline
\end{tabular}

na $=$ not applicable. $*$ Refers to use where medication was not prescribed to the individual or where medications were used in a way other than prescribed. $\uparrow$ Responses for questions on trajectory of use were incomplete; data presented here are based on the following numbers of responses for benzodiazepine use and prescription opioid use, respectively: 144 and 106 for age at first use, 70 and 91 for age when first injected, 134 and 105 for time from first use to monthly use, and 108 and 93 for time from first use to problematic use. 
3 Self-reported sources of pharmaceuticals used by study participants in the 28 days before treatment entry*

\begin{tabular}{|c|c|c|c|}
\hline & A source & $\begin{array}{l}\text { Usual } \\
\text { source }\end{array}$ & $\begin{array}{c}\text { Source for } \\
\text { most recent } \\
\text { unsanctioned use }\end{array}$ \\
\hline \multicolumn{4}{|l|}{ Benzodiazepines } \\
\hline$n$ & 144 & 123 & 139 \\
\hline Doctor - real symptom & $104(72 \%)$ & $79(64 \%)$ & - \\
\hline Doctor - fake symptom & $32(22 \%)$ & $9(7 \%)$ & - \\
\hline Any prescribed source & $113(78 \%)$ & $88(72 \%)$ & $62(45 \%)$ \\
\hline Gift & $63(44 \%)$ & $10(8 \%)$ & $50(36 \%)$ \\
\hline Swap & $22(15 \%)$ & $1(1 \%)$ & $2(1 \%)$ \\
\hline Stolen & $9(6 \%)$ & $3(2 \%)$ & $5(4 \%)$ \\
\hline Forged prescription & $5(3 \%)$ & 0 & $2(1 \%)$ \\
\hline Bought from friend & $28(19 \%)$ & $12(10 \%)$ & $11(8 \%)$ \\
\hline Buy from dealer & $25(17 \%)$ & $9(7 \%)$ & $7(5 \%)$ \\
\hline Any non-prescribed source & $80(56 \%)$ & $35(28 \%)$ & $77(55 \%)$ \\
\hline Both prescribed and non-prescribed & $80(56 \%)$ & - & - \\
\hline \multicolumn{4}{|l|}{ Prescription opioids } \\
\hline$n$ & 108 & 86 & 108 \\
\hline Doctor - real symptom & $33(31 \%)$ & $20(23 \%)$ & - \\
\hline Doctor - fake symptom & $13(12 \%)$ & $5(6 \%)$ & - \\
\hline Any prescribed source & $36(33 \%)$ & $25(29 \%)$ & $33(31 \%)$ \\
\hline Gift & $32(30 \%)$ & $10(12 \%)$ & $22(20 \%)$ \\
\hline Swap & $16(15 \%)$ & $1(1 \%)$ & $1(1 \%)$ \\
\hline Stolen & $4(4 \%)$ & 0 & 0 \\
\hline Forged prescription & $1(1 \%)$ & 0 & 0 \\
\hline Bought from friend & $42(39 \%)$ & $19(22 \%)$ & $21(19 \%)$ \\
\hline Buy from dealer & $50(46 \%)$ & $30(35 \%)$ & $29(27 \%)$ \\
\hline Any non-prescribed source & $84(78 \%)$ & $61(71 \%)$ & 75 (69\%) \\
\hline Both prescribed and non-prescribed & $20(19 \%)$ & - & - \\
\hline
\end{tabular}

tion opioids used most frequently were morphine $(65 \%, 70 / 108)$, oxycodone $(50 \%, 54 / 108)$ and methadone liquid $(29 \%, 31 / 108)$.

Injection of prescription opioids in the 28 days before treatment entry was reported by 81 participants (75\%) in the prescription opioid group; on average, they reported injecting prescription opioids on 22 of the 28 days. In contrast, injection of benzodiazepines in the 28 days before treatment entry was reported by 12 of the participants $(8 \%)$ in the benzodiazepine group; on average, they reported injecting benzodiazepines on 11 of the 28 days.

\section{Initiation and trajectories of pharmaceutical use}

On first-use occasions, both benzodiazepines and prescription opioids were most often not prescribed; $72 \%$ of participants in the benzodiazepine group and $75 \%$ of participants in the prescription opioid group reported using non-prescribed medication on the first-use occasion (Box 2). Benzodiazepine prescriptions for first-use occasions were most commonly reported to have been for anxiety $(37 \%, 15 / 41)$ and sleep $(22 \%, 9 / 41)$, and opioid prescriptions for first-use occasions were most commonly reported to have been for pain relief $(81 \%, 22 / 27)$.

Mean age at initiation of pharmaceutical use was 19.1 years for benzodiazepines and 21.2 years for prescription opioids (Box 2). The mean length of time from benzodiazepine first use to monthly use was 4.1 years and to problematic use was 6.3 years. The mean length of time from prescription opioid first use to monthly use was 3.0 years, and to problematic use was 4.4 years.

\section{Sources of pharmaceuticals}

Most benzodiazepine users $(78 \%, 113)$ reported a medical practitioner as a source of benzodiazepines in the 28 days before treatment entry (Box 3). Most also reported a medical practitioner as their usual benzodiazepine source $(72 \%, 88)$, and most reported acquiring benzodiazepines for real symptoms. Of those reporting nonmedical sources as their usual supply, access through friends or acquaintances (buying, swapping or gifts) were most common; only $7 \%$ reported buying from a dealer as their usual source.

In contrast, about half of prescription opioid users $(46 \%, 50)$ reported buying from a dealer (Box 3). Most reported using non-prescribed sources $(78 \%, 84)$, and most reported non-prescribed sources as their usual source $(71 \%, 61)$.

Few participants reported stealing or using forged prescriptions to obtain benzodiazepines and prescription opioids (Box 3), and none reported using the internet as a source.

\section{Discussion}

Our results provide information on how benzodiazepines and prescription opioids are accessed and used by people who represent the more "severe" end of the spectrum of problematic pharmaceutical drug users drug and alcohol treatment entrants reporting almost daily use of benzodiazepines or prescription opioids.

We found that pharmaceutical use was initiated early (at an average of about 20 years of age), and about three-quarters of participants reported that their initial use was via a nonmedical source. On average, it took 3-6 years for participants to progress from first use to regular or problematic use, suggesting that there is a window of opportunity for providing interventions that could avert this progression.

There were clear differences between how the two drug classes were sourced. Prescription opioids were less commonly acquired from a doctor than benzodiazepines, and most prescription opioids were acquired from non-medical sources. This is consistent with previously reported source patterns for prescription opioids. ${ }^{6,15}$ This common finding of sharing and onselling of prescription opioids has important implications for the medical profession. It highlights that while some individuals use opioids that were prescribed for other people, there may also be patients who do not use the opioids that are prescribed for them by their doctors. Most regular prescription 
opioid users reported buying their opioids from others, highlighting that this prescription drug black market is an important area to better understand. Also, prescription opioids were more likely to be injected than benzodiazepines.

In contrast to prescription opioids, a medical practitioner was the main source of benzodiazepines. As such, there may be considerable opportunities for prescriber intervention to address benzodiazepine use. This finding, coupled with the serious harms associated with benzodiazepines (in terms of acute overdose, poorer health and poorer treatment outcomes $\left.{ }^{9,10,12,13}\right)$, is interesting in light of current plans to only monitor the prescribing of S8 benzodiazepines as part of the monitoring of all S8 class drugs in Australia flunitrazepam and, from 2014, alprazolam. ${ }^{16}$ This means that, despite the greater opportunity for prescribers to intervene in benzodiazepine supply, most benzodiazepines will not fall under the current plans for prescription drug monitoring. This also represents a missed opportunity for monitoring the impact of the planned rescheduling of alprazolam on the extent of use of other, unmonitored, benzodiazepines.

In contrast, monitoring opioid prescribing is unlikely to have a substantial impact on the behaviour of prescription opioid users, as they mostly acquire prescription opioids from non-medical sources. Also, it might give prescribers false confidence if they relied on the information from monitoring systems for the clinical assessment of drug-seeking behaviour alone, as opioids acquired from non-medical sources would not appear in such systems.

Other strategies - such as education of consumers and health professionals, and expansion of nonpharmaceutical evidence-based treatments for chronic pain and mental health disorders - are crucial. Approaches such as "universal precautions" with opioid prescribing have been recommended internationally ${ }^{18}$ and in Australian pol${ }_{\text {icy, }}{ }^{19}$ but are yet to be established in practice.

Education for consumers on the harms associated with sharing medi- cations is vital. Benzodiazepines are commonly implicated in non-fatal ${ }^{12}$ and fatal ${ }^{13,20}$ opioid overdose. We found that one-third of participants were given benzodiazepines as a gift for their most recent unsanctioned use. On first-use occasions, only onequarter of benzodiazepines and prescription opioids were from a prescriber. Education about risks associated with self-diagnosis, peer diagnosis and self-medicating may help reduce medication sharing. Also, education initiatives for consumers could be used to challenge the perception that benzodiazepines are "less risky" 21 than illicit drugs.

Our study has strengths and weaknesses. Convenience sampling and collection of self-reported data are well recognised and valid methods for studying individuals who engage in unsanctioned substance use $\mathrm{e}^{22,23}$ and provide reliable answers in this context. ${ }^{23}$ However, it is well recognised that these methods affect the accuracy and representativeness of the data collected, compared with prospectively collected and objectively validated data from random or more generalisable samples.

The participants of our study had similar demographic characteristics to participants in other studies of drug treatment clients in Australia conducted in Australia. ${ }^{7,24}$ Although they may not represent most long-term problematic users of pharmaceuticals in the broader community, they represent a group who are more likely to experience acute harms from their pharmaceutical use and who use larger amounts of pharmaceuticals. As such, they are a priority when targeting strategies aimed at minimising pharmaceutical misuse.

These findings show that different approaches may be required to reduce unsanctioned use of different pharmaceuticals. Prescription drug monitoring programs need to ensure that the right substances are monitored. Current proposals may represent a significant lost opportunity with regard to the most benzodiazepines. Finally, developing guidelines and resources that help health professionals respond to pharmaceutical misuse identified through monitoring systems will maximise opportunities to reduce harm.
Acknowledgements: Suzanne Nielsen and Louisa Degenhardt are current recipients of NHMRC Fellowships. The larger study that this work was drawn from was commissioned by the Ministerial Council on Drug Strategy through the Cost Shared Funding Model with the Victorian Department of Human Services. The funders had no role in the design of the study, analysis of the data or preparation of the manuscript.

Competing interests: Suzanne Nielsen, Louisa Degenhardt and Nicholas Lintzeris has been investigators on untied education grants, unrelated to this work, from Reckitt Benckiser (who make buprenorphine and a buprenorphine-naloxone combination, which are used in the treatment of opioid dependence). Nicholas Lintzeris has received honoraria to present at professional development courses. Reckitt Benckiser had no role in or knowledge of this study.

Received 31 Aug 2012, accepted 14 Sep 2013.

1 Leong M, Murnion B, Haber PS. Examination of opioid prescribing in Australia from 1992 to 2007. Intern Med J 2009; 39: 676-681.

2 Rintoul AC, Dobbin MDH, Drummer OH, Ozanne-Smith J. Increasing deaths involving oxycodone, Victoria, Australia, 2000-09. Inj Prev 2010; 17: 254-259.

3 Executive Office of the President of the United States. Epidemic: responding to America's prescription drug abuse crisis. 2011. http://www.whitehouse.gov/ sites/default/files/ondcp/policy-and-research/rx_abuse_plan.pdf (accessed Aug 2012).

4 Maxwell JC. The prescription drug epidemic in the United States: a perfect storm. Drug Alcohol Rev 2011; 30: 264-270.

5 Nielsen S, Dietze P, Lee N, et al. Concurrent buprenorphine and benzodiazepines use and self-reported opioid toxicity in opioid substitution treatment. Addiction 2007; 102: 616-622.

6 Rosenblum A, Parrino M, Schnoll SH, et al. Prescription opioid abuse among enrollees into methadone maintenance treatment. Drug Alcohol Depend 2007; 90: 64-71.

7 Ross J, Teesson M, Darke S, et al. The characteristics of heroin users entering treatment: findings from the Australian treatment outcome study (ATOS). Drug Alcohol Rev 2005; 24: 411-418.

8 Lintzeris N, Nielsen S. Benzodiazepines, methadone, buprenorphine: interactions and clinical management. Am J Addict 2010; 19: 59-72.

9 Brands B, Blake J, Marsh DC, et al. The impact of benzodiazepine use on methadone maintenance treatment outcomes. J Addict Dis 2008; 27: 37-48.

10 Darke S, Hall W, Ross M, Wodak A. Benzodiazepine use and HIV risk-taking behaviour among injecting drug users. Drug Alcohol Depend 1992; 31: 31-36.

11 Brands B, Blake J, Sproule B, et al. Prescription opioid abuse in patients presenting for methadone maintenance treatment. Drug Alcohol Depend 2004; 73: 199-207.

12 Dietze P, Jolley D, Fry C, Bammer G. Transient changes in behaviour lead to heroin overdose: results from a case-crossover study of non-fatal overdose. Addiction 2005; 100:636-642

13 Caplehorn JR, Drummer OH. Fatal methadone toxicity: signs and circumstances, and the role of benzodiazepines. Aust N Z J Public Health 2002; 26: 358-362.

14 Martyres RF, Clode D, Burns JM. Seeking drugs or seeking help? Escalating "doctor shopping" by young heroin users before fatal overdose. Med J Aust 2004; 180: $211-214$

15 Substance Abuse and Mental Health Services Administration. Results from the 2010 National Survey on Drug Use and Health: summary of national findings. Rockville, Md: SAMHSA, 2011. (NSDUH Series H-41, HHS Publication No. (SMA) 11-4658.) http://www.samhsa.gov/data/nsduh/2k10nsduh/2k10results.htm (accessed Aug 2012).

16 Shand FL, Campbell G, Hall W, et al. Real-time monitoring of Schedule 8 medicines in Australia. Med J Aust 2013; 198: 80-81.

17 Nielsen S, Bruno R, Carruthers S, et al. Investigation of pharmaceutical misuse amongst drug treatment clients. Melbourne: Turning Point Alcohol and Drug Centre, 2008.

18 Gourlay DL, Heit HA, Almahrezi A. Universal precautions in pain medicine: a rational approach to the treatment of chronic pain. Pain Med 2005; 6: 107-112.

19 Royal Australasian College of Physicians. Prescription opioid policy: improving management of chronic non-malignant pain and prevention of problems associated with prescription opioid use. Sydney: RACP, 2009.

20 Ernst E, Bartu A, Popescu A, et al. Methadone-related deaths in Western Australia 1993-99. Aust N Z J Public Health 2002; 26: 364-370.

21 Fountain J, Griffiths P, Farrell M, et al. Benzodiazepines in polydrug-using repertoires: the impact of the decreased availability of temazepam gel-filled capsules. Drugs Educ Prev Policy 1999; 6: 61-69.

22 Shand F, Topp L, Darke S, et al. The monitoring of drug trends in Australia. Drug Alcohol Rev 2003; 22: 61-72.

23 Darke S. Self-report among injecting drug users: a review. Drug Alcohol Depend 1998; 51: 253-263.

24 Darke S, Ross J, Teesson M, Lynskey M. Health service utilization and benzodiazepine use among heroin users: findings from the Australian Treatment Outcome Study (ATOS). Addiction 2003; 98:1129-1135. 